\title{
Armut und Autobiographie - Versuch einer Begriffsanalyse
}

\author{
Małgorzata Bogaczyk-Vormayr (Adam Mickiewicz-Universität Poznań, z.Z. \\ Universität Salzburg)
}

„Nie werden wir mit dem fertig, was wir Zufall nennen”, schrieb Wilhelm Dilthey in seinen bedeutsamen Erläuterungen zur Selbstbiografie, die für die gegenwärtigen humanwissenschaftlichen Auffassungen von Lebensverlauf, Zeitbewusstsein und Selbstbesinnung als klassische Quelle gelten. Der Grundbegriff der Dilthey'schen Analyse ist der Zusammenhang des Lebens, seine Hauptfrage bezieht sich auf das Phänomen des Zusammenhangs - das Verständnis des Zusammenhangs von einzelnen Erlebnissen. Der Zusammenhang des Lebens wird sich präsentieren bzw. wird von dem sich beobachtenden Subjekt präsent gemacht, in einem für jeden Menschen universellen Bedürfnis nach Selbstbiographie. $\mathrm{Zu}$ einer solchen narrativen Suche und einer Klärung des Zusammenhangs des Lebens schrieb Dilthey:

\begin{abstract}
Die Einheiten sind in den Konzeptionen von Erlebnissen gebildet, in denen Gegenwärtiges und Vergangenes durch eine gemeinsame Bedeutung zusammengehalten ist. Unter diesen Erlebnissen sind diejenigen, die für sich und den Zusammenhang des Lebens eine besondere Dignität haben, in der Erinnerung bewahrt und aus dem endlosen Fluß des Geschehens und Vergessens herausgehoben; und ein Zusammenhang ist im Leben selber gebildet worden, von verschiedenen Standorten desselben aus, in beständigen Verschiebungen. Da ist also das Geschäft historischer Darstellung schon durch das Leben selber halb getan. Einheiten sind als Erlebnisse geformt; aus der endlosen, zahllosen Vielheit ist eine Auswahl dessen vorbereitet, was darstellungswürdig ist (Dilthey 1927, 202).
\end{abstract}

Der Charakter des Lebensverlaufs ist durch die Beziehungen zwischen Gegenwart, Vergangenheit und Zukunft bestimmt (Dilthey 1927, 72) und die Selbstbiographie ist nach Dilthey eine Deutung der Verbindung von Zufall, Schicksal und Charakter (74). Ist ein Leben ohne Selbstbiographie überhaupt möglich? Leben und Selbstbiographie sind zwei kongruente Entitäten - das autobiographische Bewusstsein, der Ich-Inhalt, welcher „das Leben“ als „das Eigene“, als „das Erlebte“ darstellt. Die Selbstbiographie ist eine Form des Gedächtnisses, eine Erinnerungs- und Identitätsarbeit. Als solche zeichnet sie sich u.a. dadurch aus, dass sie als eine Interpretation des Ich fungiert, in welcher unser Bedürfnis nach Ausgrenzung, Ausklammerung, nach 
Verschweigen der für uns erschütternden, uns erniedrigenden Erlebnisse zur Geltung kommt. Zu einem solchen Teil der Selbstbiographie kann die Erfahrung von Armut gehören: Ich möchte hier kurz aufzeigen, dass sich die Armut im autobiographischen Erzählen nicht verleugnen lässt und dass genau jene Autobiographien, die von der Armut berichten, den wahren Ereignissen (d.h. der Selbstbiographie) möglichst nahe bleiben. Armut bildet ein den Menschen zutiefst als Person konstruierendes und dekonstruierendes Element, so dass die Armutserfahrung, wenn diese auftritt, selbst zum Zusammenhang des Lebens wird.

In einer gewissen Polemik gegenüber Dilthey stelle ich hier folgende Arbeitshypothesen auf: (1) Die Selbstbiographie dient dazu, die Zusammenhänge des Lebens $\mathrm{zu}$ verstehen, nie jedoch dazu, diese $\mathrm{zu}$ konstruieren; (2) Die Autobiographie - als eine nach außen gerichtete Aussage des Selbst - hat ihren Kern in der Selbstbiographie, jedoch dienen die narrativen Elemente der Autobiographie vor allem einer Geschichte, einer Darstellung, und nicht dem Biographischen selbst; ergo (3) Nur eine Autobiographie, in welcher man versucht der Selbstbiographie treu zu bleiben, kann die Leserschaft mit ihrer Authentizität überzeugen und gleichzeitig literarisch gelungen sein. In der Weltliteratur haben wir viele bedeutende Porträts von Menschen, die in großer Armut aufwachsen und aus dieser herauswachsen - bei Nikolaj Gogol, Victor Hugo, Charles Dickens, Knut Hamsun, Joseph Conrad, John Steinbeck und vielen anderen. Das "Herauswachsen" meint hier eine Entwicklung zur geistigen Unabhängigkeit von den erlebten Erniedrigungen, es bedeutet eine Bewältigung des vermuteten "Fatums", eines schon am Beginn des Lebens uns zugeschriebenen Lebenslaufes. In diesem Herauswachsen wird man doch fertig mit dem sog. Zufall, man hat nämlich den Zusammenhang des Lebens tatsächlich aus der Wahrheit über das eigene Leben und aus der eigenen Wirkungskraft herausgelöst. Die Autobiographien, welche diese Aufgabe erfüllen, werden zu großen, universell verstandenen Geschichten - so sah es auch Dilthey:

Die Macht und Breite des eigenen Lebens, die Energie der Besinnung über dasselbe ist die Grundlage des geschichtlichen Sehens. Sie allein ermöglicht, den blutlosen Schatten des Vergangenen ein zweites Leben zu geben. Ihre Verbindung mit einem grenzenlosen Bedürfnis, sich fremdem Dasein hinzugeben, sein eigenes Selbst in diesem zu verlieren, macht den großen Geschichtsschreiber (202).

Es handelt sich also um Geschichten, in denen das erzählende Ich bereit ist, reif dafür ist, den ersten Platz auf der Geschichtenbühne dem Thema einzuräumen, und nicht über sich zu jammern oder sich selbst zu loben, sondern die Phänomene, die Ereignisse einem anderen zu erzählen. Eine wunderbare Illustration dazu bildet der Roman Conversazione in Sicilia von Elio Vittorini aus dem Jahre 1939. Vittorini beteuerte des Öfteren, dieser 
Roman sei keine Autobiographie, aber ich meine nur, dass diese Geschichte ebenso gut von jedem jungen Italiener aus dieser Zeit, an diesem Ort handeln könnte; er betonte, es sei ein Roman, in dem er als Schriftsteller es geschafft hätte, etwas Wahres auszusprechen. Die Reise eines jungen Mannes in sein Heimatdorf, in das arme Sizilien, wo Hunger und Krankheit herrschen, ist gleichzeitig ein trauriges Bildnis von den falschen Hoffnungen einer Nation wie auch ein erhellendes Gemälde von der Würde des einzelnen Menschen. Die von großer Armut betroffene Region ist ebenso ein Thema dieses Buches wie die Rückkehr des Protagonisten nach Hause und dessen Selbstreflexion. Sein kurzer Gang durch die Grotten, welche von ärmsten Kranken bewohnt werden, ist ein realistisches Armutsbild, das uns davon überzeugt, dass die Poetisierung oder das Verfeinern von zutiefst bedrohlichen Lebenserfahrungen die Betroffenen, anstatt sie vielleicht zu schützen, viel eher verletzen würde. Eine solche Literarisierung degradiert die Menschen zu Objekten. Das Dialogische steht in Vittorinis Roman im Mittelpunkt: Der Roman besteht aus vielen kurzen Begegnungen und Gesprächen mit unbekannten oder aus der Vergangenheit aufgetauchten Menschen. Aus diesen Gesprächen, die u.a. eine Landschaft der Armut schildern, formt sich langsam der Zusammenhang des Lebens.

So wie Conversazione in Sicilia ein gelungenes Beispiel für ein selbstbiographisches Bemühen darstellt, sind weitere drei Titel, die ich im nächsten Schritt nennen möchte, Beispiele für eine Autobiographie. Ich verweise deshalb auf diese drei Werke, weil deren literarische Qualität den Ansprüchen einer Selbstbiographie exakt entspricht und somit meine dritte Arbeitshypothese illustrieren. Indem alle drei Bücher dasselbe Sujet thematisieren - das Leben im KZ/Gulag/Lager - und sich durch alle derselbe rote Faden zieht, nämlich der Hunger, unterscheiden sie sich dennoch voneinander - und das macht einen Vergleich so spannend - in der Erzählweise. Und so wird ...trotzdem Ja zum Leben sagen von Victor Frankl, ein autobiografischer Bericht aus Auschwitz, sofort nach der Befreiung 1945 niedergeschrieben; Der blaue Schnee ist ein (auto)biographischer Roman von Piotr Bednarski, veröffentlicht 1996, einige Jahrzehnte später, nachdem Bednarski als Kind den sibirischen Gulag verlassen hat; und der Roman Atemschaukel von Herta Müller aus dem Jahre 2009 verharrt in einer rein literarischen Form, der Inhalt des Buches basiert jedoch zum Großteil auf den Gesprächen der Autorin mit Überlebenden, vor allem mit Oskar Pastior, geführt in den ersten Jahren des 21. Jahrhunderts - und so wird diese Geschichte des Lebens in einem sowjetischen Lager von einem männlichen Ich namens Leopold Auberg berichtet.

Frankl schreibt nur von Erfahrungen, die für ihn etwas völlig Neues darstellen - von der gänzlich neuen Bedeutung der sozialen Beziehungen, der alltäglichen Bedürfnisse, von einem bis dahin ihm unbekannten Leiden. Die sog. Flucht nach innen beschreibt er mit der Präzision seines Faches - 
psychologisch: Er berichtet über die eigene Flucht und stellt gleichzeitig die Diagnose, dass diese Methode das Geistige, z.B. das Gedächtnis eines Menschen tatsächlich in Schutz nehmen kann. Eine weitere interessante Diagnose bezieht sich auf die Phase der Apathie, die innerhalb jeder Krisensituation auftritt. Es scheint eine Flucht vor den erfahrenen Verletzungen und vor den eigenen Hoffnungen zu sein. $\mathrm{Zu}$ den Phasen und Methoden der Apathie-Bewältigung zählt die selbstbiographische Arbeit, u.a. die mündlichen und schriftlichen Berichte.

Wenden wir uns also dem Thema des Hungers zu: Bei Frankl als eine neue Erfahrung und bei Bednarski, ganz konträr, als ein Lebenszustand beschrieben:

Die denkbar höchstgradige Unterernährung, unter der die Häftlinge zu leiden hatten, läßt es selbstverständlich erscheinen, daß innerhalb der primitiven Triebhaftigkeit, zu der das seelische Leben im Lager 'regrediert', der Nahrungstrieb im Mittelpunkt steht. Beobachten wir einmal das Gros der Häftlinge, wenn sie auf dem Arbeitsplatz beisammenstehen und gerade nicht scharf beaufsichtigt werden. Sogleich werden sie vom Essen zu reden beginnen! (Frankl 2003, 53).

Wir waren immer hungrig, zerlumpt und verlaust. ... Gewöhnlich nahmen wir unsere Armut und den Tod um uns herum nicht wahr. Das war unsere Welt, unsere Wirklichkeit, unser Alltag.

Im Unterricht fragte man uns wieder einmal, was wir später werden wollen. Unsere Jungenbande war die Sturmgruppe; jeder von uns wollte entweder Seemann oder Flieger werden. ... Diesmal wurde Sascha Swjerdlow aufgerufen. Er antwortete nicht wie gewohnt und überraschte uns, brachte uns auf den Boden zurück. Er erklärte ganz einfach, dass er später am liebsten ein Laib Brot wäre, weil ein Brot nie hungrig sei und jeder sich nach Brot sehnen würde. Durch unsere Gruppe ging ein Raunen. Alle schauten zu Sascha wie zu einem Verräter hinüber und warfen ihm insgeheim vor, dass er aussprach, was wir zu verbergen suchten. Jeder von uns dachte doch ständig an Brot und sehnte sich danach, genug davon zu haben. Unsere Träume bestanden aus Brot (Bednarski 2007, 5, 22-23).

Der Blaue Schnee widmet sich noch einigen Themen, die ich als repräsentative Motive des autobiographischen Erzählens von Armutserfahrungen ansehe, diese sind: (i) Liebe zu einem schönen, über die alltägliche Hässlichkeit erhabenes Objekt (hier ist es die Mutter des Erzählers, „Schönheit“ genannt), (ii) Freundschaftsbeziehungen als Bündnis von Menschen in ähnlichen Lebenslagen, (iii) geistige Entwicklung als Chance zur Unabhängigkeit (hier sind es u.a. Religiosität und schriftstellerisches Talent). Diese Motive finden wir auch in dem genialen „Hunger-Roman“ Atemschaukel, ebenso wie die Motive der Apathie und der Flucht nach innen. Der Hunger liefert den Grund dafür, doch den Kampf aufzunehmen: Man bekämpft die Angst und die Scham, 
um das schlimmste und gewöhnlichste Zeichen der eigenen, herabwürdigenden Lage zu bekämpfen, eben wiederum den Hunger:

Und es kommt der Abend. Und alle kommen von der Arbeit heim. Und alle steigen in den Hunger. Es ist ein Bettgestell, wenn ein Hungriger den anderen Hungrigen zuschaut. Aber das täuscht, ich spüre an mir, der Hunger steigt in uns hinein. Wir sind das Gestell für den Hunger. Wir alle essen mit geschlossenen Augen. Wir füttern den Hunger die ganze Nacht. Wir mästen ihn hoch auf die Schaufel. Ich esse einen kurzen Schlaf, dann wache ich auf und esse den nächsten kurzen Schlaf. Ein Traum ist wie der andere, es wird gegessen (Müller 2009, 89).

Die vom Roman-Protagonisten erfundenen Figuren des Hungers sind seine Begleiter im Lager: der Hungerengel, das Brotgericht, die Brot-Dreifaltigkeit. Der Hunger erfüllt das gesamte Lager: „Sogar der Wind fütterte den Hunger, er webte sichtbares Essen, überhaupt nicht abstrakt (Müller, 159).” Man kämpft, damit dieser nicht die Seele/Psyche insgesamt ausfüllt, einen in eine unheilbare Krankheit zwingt. Die erfundenen Figuren seines Schreckens verlassen Leopold Auberg aber nicht, nachdem er das Lager verlässt und nach Hause zurückkehrt. Dies ist wiederum ein markantes Motiv im autobiographischen Erzählen von den schwierigsten Lebenskrisen: Einen Teil des erlebten Schreckens trägt man in das spätere Leben mit, und das Gefühl der Entfremdung, in welche man durch dieses Unglück geraten ist, wird pfleglich aufrecht erhalten, zum eigenen Schaden:

Mein gründliches Imstichlassen. Ich brauche viel Nähe, aber ich gebe mich nicht aus der Hand. Ich beherrsche das seidene Lächeln im Zurückweichen. Seit dem Hungerengel erlaube ich niemandem, mich zu besitzen (Müller, 295).

Armut liefert einen starken Grund dafür, sich unter anderen fremd zu fühlen, die erlebte Ausgrenzung wirkt in der Folge als Schutzfaktor, ständig aktiv in dem „neuen“, posttraumatischen Leben. Viele Beobachtungen dieser Art machten auch noch drei weitere Autoren, die ich abschließend erwähnen möchte - alle mit ganz anderen Biographien als die Autoren/Helden der schon oben genannten Titel. Dennoch bildet auch bei ihnen die Armut ein zentrales Motiv ihres literarischen Schaffens: J. M.Coetzee, Karl-Markus Gauß und Aglaja Veteranyi.

J. M. Coetzee berichtet in seinen autobiographischen Romanen über die von ihm zwar nicht miterlebte, aber in seiner Welt stets präsente Armut. In Boyhood: Scenes from Provincial Life begleitet immer eine Schar schwarzer Kinder die Weißen, die auf dem Weg zur Schule sind, auf dem Tennisplatz spielen, im Kaffeehaus sitzen. Die kleine Worcester-Welt taucht in den Kindheitserinnerungen des Schriftstellers wie ein großer Schauplatz der Apartheid auf. Im Kleinen und Privaten, im authentisch von Coetzee Erlebten, spiegelt sich die Wahrheit über die Unterdrückung und Armut der anderen. Es 
scheint so, dass erst diese schriftstellerische Erinnerungsarbeit die Botschaft des Romans zutage bringt, nämlich dass man den Zusammenhang des eigenen Lebens erst gewinnt, wenn man es unter einem Ganzen sieht, unter dem, was vorher unterdrückt, ausgegrenzt wurde. Die Armut der anderen beeinflusst auch das eigene Leben, unsere Sichtweise, unseren Lebensgang.

Auch Karl-Markus Gauß formuliert gekonnt, wenn er davon spricht, dass ein Ort nicht durch Armut, Arbeitslosigkeit, Gewalt und Verfall zu einem Armuts- und Ausgrenzungsort, also zu einem Slum wird, sondern durch seine Unsichtbarkeit. Die Reportagen aus seinen Reisen durch Ost- und Südeuropa bekommt die Leserschaft als oral history, als biographische und autobiographische Aufzeichnungen vorgeführt. Bei ethnischen Minderheiten und in Romasiedlungen - inmitten einer europäischen Großstadt, oft in einem Land an der EU-Grenze - trifft Grauß immer wieder auf Orte einer „Vollständigen, alle Lebensbereiche erfassenden Apartheid“ (Grauß 2006, 97). Die Ghettos entstehen aus Arbeitslosigkeit und Gewalt, jedoch die Unsichtbarkeit - „Der Slum ist nebenan, aber man sieht ihn nicht“ (22) -, also die unsichtbare Nachbarschaft, ist das Wesen eines Ghettos, das Wesen der Armut.

Eine zu Coetzee konträre Perspektive bieten die autobiographischen Bücher der rumänisch-schweizerischen Autorin Aglaja Veteranyi, die tatsächlich eine Kindheit in völliger Armut durchlebte. Veteranyi scheint aus ihrer Vergangenheit eines Flüchtlingskindes in die Welt der Erfolgreichen, Gebildeten und Sicheren zu entkommen, findet sich in dieser jedoch zeit ihres Lebens nie wirklich zurecht. Ihr literarisches Werk wird von einem Thema dominiert: von ihrer schwierigen, u.a. durch Armut gekennzeichneten Kindheit widmete:

\section{DIE FLUCHT}

Das Kind packt die Puppe in den Koffer.

Die Mutter packt das Kind in den Koffer.

Der Vater packt Mutter und Haus in den Koffer.

Das Ausland packt Vater mit Koffer in den Koffer.

Schickt alles zurück.

Es verstecken sich im Wald:

1 Puppe

1 Kind

1 Mutter

1 Vater

1 Haus

2 Koffer

1 Flucht 
Veteranyi war bis zum 20. Lebensjahr eine Analphabetin, das Schreiben erhielt für sie dadurch einen Sinn, dass sie sich damit selbst enthüllen konnte. Obwohl ihr großer Bühnen- und Schreib-Erfolg in Wirklichkeit nur am Rande ihrer traumatischen Erinnerungen stattfand, zeigt dies auf, wie die innere und sodann äußere Unabhängigkeit mit dem Grad der Bildung in Zusammenhang stehen.

Ich versuchte aufzuzeigen, wie die durch Armut gekennzeichnete Autobiographie ihren Zusammenhang entdecken bzw. konstituieren kann, indem diese zutiefst verletzenden Erlebnisse nicht ausgeblendet, sondern durch die Tätigkeit des Berichtens bearbeitet werden. So formt sich meine dritte Arbeitshypothese zu einer Conclusio: Das autobiographische Erzählen, um mit seiner Authentizität und seiner literarischen Qualität zu überzeugen, muss der Selbstbiographie treu bleiben.

\section{Literatur:}

Bednarski, P. 2007. Der blaue Schnee. Übers. von J. Manc. Berlin: List Verlag.

Coetzee, J. M. 1997. Boyhood: Scenes from Provincial Life. London: Secker \& Warburg.

Dilthey, W. 1927. Der Aufbau der geschichtlichen Welt in den Geisteswissenschaften. Leipzig: Teubner.

Frankl, V. E. 2003. ...trotzdem Ja zum Leben sagen. Ein Psychologe erlebt das Konzentrationslager. München: Deutscher Taschenbuch Verlag.

Gauß, K.-M. 2006. Die Hundeesser von Svinia. München: Deutscher Taschenbuch Verlag.

Müller, H. 2009. Atemschaukel. München: Carl Hanser Verlag.

Veteranyi, A. 2004. Vom geräumten Meer, den gemieteten Socken und Frau Butter. Geschichten. München: Deutsche Verlags-Anstalt.

Vittorini, E. 2006. Conversazione in Sicilia. Milano: Biblioteca Univ. Rizzoli. 


\title{
Małgorzata Bogaczyk-Vormayr (Poznań, Salzburg)
}

Poverty and Autobiography: An Attempt of Concept Analysis.

\begin{abstract}
This short working paper is my first attempt to present my concept analysis of relation between the poverty experiences - e.g. childhood suffering by war and migration background, daily life suffering by starvation, abuse, racism etc. - and the process of self-understanding and resilience with the help of an oral history or literature (non-fiction as much as fiction novels). I reflect Wilhelm Dilthey's opinion about the distinction between autobiography and Self-biography, and I present the Self-biography as a right way to concretize the themes of poverty and exclusion.
\end{abstract}

Keywords: Poverty, hunger, autobiography, self, literature and crisis

Doi: $10.14746 /$ eip.2014.2.6 